\title{
Using Sulcal Basins for Analyzing Functional Activations Patterns in the Human Brain
}

\author{
G. Lohmann and D.Y. von Cramon \\ Max-Planck-Institute of Cognitive Neuroscience \\ Stephanstr. 1a, 04103 Leipzig, Germany \\ Ph: ++49-341-9940 217, Fax: ++49-341-9940 221 \\ \{lohmann, cramon\}@cns.mpg.de
}

\begin{abstract}
In previous work, we have introduced the concept of sulcal basins. Sulcal basins are subdivisions of cortical folds that allow to establish a complete parcellation of the cortical surface into separate regions. In this paper, we present methods of using this concept to support the analysis of functional activation patterns in the human brain as measured by fMRI (functional magnetic resonance imaging) experiments. In particular, we present two methods of performing inter-subject averages. The first method uses a form of non-linear spatial normalization based on sulcal basin landmarks. The second method performs group averages using sulcal basins themselves as entities for averaging. This second approach has the advantage of ensuring that truly anatomically homologue entities enter the averaging process. In addition, it yields results that are immediately interpretable by a specialist.

The methods are presented in the context of an fMRI experiment in which 10 test subjects were asked to respond to various visual stimuli.
\end{abstract}

\section{Introduction}

In previous work, we have introduced the concept of sulcal basins [7],[5],[6]. Sulcal basins are subdivisions of cortical folds that allow to establish a complete parcellation of the cortical surface into separate regions. These regions are neuroanatomically meaningful and can be identified from MR data sets across many subjects.

In this paper, we present methods of using this concept to support the analysis of functional activation patterns in the human brain as measured by fMRI (functional magnetic resonance imaging) experiments. FMRI allows to create digital images that display local changes in blood flow with a spatial resolution of about $3 \mathrm{~mm}$ and a temporal resolution of up to 1 second. Since its invention a few years ago [1], it has become one of the most important technologies used in human brain mapping research.

The data produced by a typical fMRI experiment consist of a time sequence of digital images taken every $n$ seconds. Each image contains several 2D slices where typically slices are about $5 \mathrm{~mm}$ thick and gaps between slices are about $2 \mathrm{~mm}$ wide. Usually, two or more experimental conditions are alternated within the 
same experiment. For instance, some baseline condition may be contrasted with a condition in which some visual or auditory stimulus is presented.

The aim of fMRI data analysis is threefold. The first aim is to detect image regions which display a significant difference in image intensity between various experimental conditions. We will refer to those regions as "activation areas". Secondly, the exact anatomical location of activation areas should be reported. And thirdly, some method of inter-subject averaging must be provided. At present, inter-subject averaging is usually performed by applying some form of spatial normalization to each individual data set using image warping techniques, and then computing pixel-wise averages in a stereotactic coordinate space [13].

In this paper, we will propose a method of inter-subject averaging that does not require spatial normalizations and warping. In fact, the sulcal basin model provides a means of performing inter-subject comparisons and group averages based on individual anatomy rather than on a stereotactic coordinate space.

However, if needed, the sulcal basin model can also used to perform a spatial normalization based on non-linear warping. In this paper, we will present both methods of inter-subject averaging. We present the warping method primarily for the purpose of validating the basin concept.

\section{2 fMRI Data Analysis}

\subsection{Experimental Data}

We will describe our methods in the context of an fMRI experiment described by Pollmann et al. [14]. In this experiment, 10 healthy test subjects were asked to respond to various visual stimuli while fMRI data were being recorded.

Sixteen fMRI slices with a thickness of $5 \mathrm{~mm}$, interslice distance $2 \mathrm{~mm}, 19,2 \mathrm{~cm}$ FOV and an image matrix of $64 \times 64$ were collected at a 3T Bruker 30/100 Medspec (Bruker Medizintechnik GmbH, Ettlingen, Germany) using a gradient recalled EPI sequence $(\mathrm{TR}=2000 \mathrm{~ms}, \mathrm{TE}=40 \mathrm{~ms}$, flip angle=40). All 16 slices were recorded every 2 seconds. During the experiment, a baseline condition and an experimental condition alternated. At the same time, sixteen anatomical T1weighted $2 \mathrm{D}$ slices were also recorded that were geometrically aligned with the functional data.

In addition to the fMRI data, we also obtained anatomical 3D MR data sets from all 10 subjects. The spatial resolution between planes was approx. $1.5 \mathrm{~mm}$ and the within-plane resolution was set to approx. $0.95 \mathrm{~mm} \times 0.95 \mathrm{~mm}$. The images were resampled to obtain isotropic voxels of size $1 \mathrm{~mm} \times 1 \mathrm{~mm} \times 1 \mathrm{~mm}$ so that each data set contained 160 slices with $200 \times 160$ pixels in each slice. All $3 \mathrm{D}$ data sets were rotated into a stereotactic coordinate system such that the origin was halfway between CA and CP (see also [9]).

\subsection{Statistical Analysis}

The analysis of the fMRI data proceeds in several steps [8]. After some preprocessing involving radiometric and geometric corrections, a statistical t-test is 
employed in order to identify image regions that show a significant difference in image intensities between the experimental conditions.

The result of this analysis is a map in which the fMRI image sequence is collapsed into one image where each pixel encodes the degree of significance with which the image intensity differs between the experimental conditions. The degree of significance is represented by $p$-values that are given as normalized $z$ scores. Therefore, these maps are often called a "zmap". We obtained such a zmap for each test subject.

\subsection{Spatial Normalization}

In order to facilitate group studies, the zmaps from various test subjects must be geometrically aligned in 3D space. This is achieved in two steps: co-registration and spatial normalization.

During co-registration the zmaps are rotated and shifted into correspondence with the 3D anatomical MR data set acquired from each subject. Remember that the $3 \mathrm{D}$ anatomical data sets are already rotated and translated into a common stereotactic coordinate system. Thus, co-registration also aligns the zmaps.

The co-registration was achieved as follows. As noted before, during the experimental session we recorded not only functional MR slices but also 2D anatomical MR slices that were geometrically aligned with the functional data. We used these antomical slices to compute rotational and translational parameters that maximized the correlation between the anatomical 2D data slices and the $3 \mathrm{D}$ reference data set. We then used these same parameters to register the zmaps as well. The co-registration algorithm is explained in more detail in [11].

After co-registration, all zmaps reside in a common stereotactic coordinate system. However, due to differences in individual anatomy, this does not guarantee that corresponding anatomical locations of different subjects occupy the same location within this coordinate system. Therefore, some form of spatial normalization is required.

Spatial normalization is frequently used in the context of human brain mapping in an effort to remove inter-subject or inter-modal variability. Generally, the aim is to geometrically align one data set with another such that corresponding brain locations are mapped onto each other and spatial variability is diminished([3],[13]).

The simplest form of spatial normalization is a linear scaling that brings all data sets into a common standard size. More sophisticated methods involve non-linear warping techniques that seek to warp individual data sets onto some reference atlas. Spatial normalization approaches can be loosely classified into two major groups: intensity-driven and landmark-driven approaches. Intensity driven approaches try to match locally corresponding image regions of similar grey value intensity (e.g. [2],[4],[10],[18]). Landmark-driven approaches use anatomical landmarks such as curves[17] or surfaces [16][19] to guide the warping. For a complete reference on spatial normalization and registration see [20],[12].

In the following, we will propose a new method of spatial normalization based on the concept of sulcal basins. 

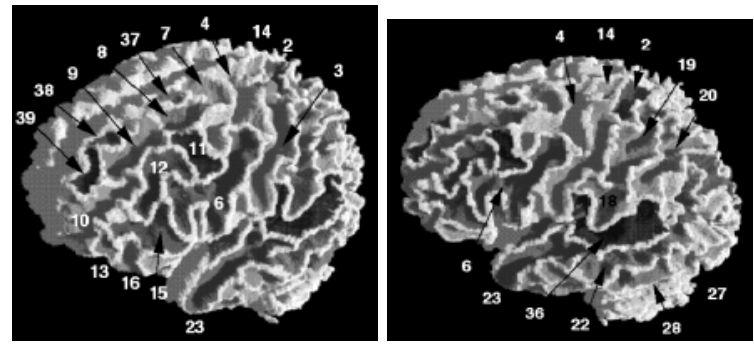

Fig. 1. The result of a segmentation and labelling of sulcal basins. The numbers indicate neuroanatomical labels, e.g. '3' is the label for the inferior part of the central sulcus. The anatomical model contains 38 left-hemispheric basins.

\section{Sulcal Basins}

Sulcal basins are concavities on top of the white matter surface (after removal of the cortical compartment) that are bounded by convex ridges that separate one basin from the next so that adjacent sulcal basins meet at the top of the ridge. The entire white matter surface is covered by such concavities so that a decomposition into sulcal basins yields a complete parcellation of the surface.

A method for segmenting and labelling sulcal basins was introduced in [7]. Figure 1 shows the resulting labelled basins. The numbers indicate neuroanatomical labels. Our present model contains 38 left-hemispheric basins.

The sulcal basin model facilitates a new approach to non-linear spatial normalization. The basic idea is to guide a surface based warping mechanism by sulcal basin landmarks.

The surface to be warped is the morphological closure of the white matter which is obtained by applying a 3D morphological closing filter to the white matter image. The surface to be warped is the surface of the morphological closure (figure 2c).

To normalize brain shapes, we first selected a "model" brain from our 10 data sets to which all other brains were subsequently deformed. All data sets were initially subjected to a linear scaling in the $x, y$ and $z$-directions so that they all had the same bounding box. We then use a quadratic polynomial $F$ to perform an additional non-linear deformation that uses sulcal basin information. Note that all voxels belonging to the surface of the morphological closure are also elements of some sulcal basin so that every surface voxel inherits a basin label from the basin to which it belongs. The quadratic polynomial $F$ has the form:

$$
F(x, y, z)=\left(\begin{array}{l}
a_{00}+a_{01} x+a_{02} y+a_{03} z+a_{04} x y+a_{05} x z+a_{06} y z+a_{07} x^{2}+a_{08} y^{2}+a_{09} z^{2} \\
a_{10}+a_{11} x+a_{12} y+a_{13} z+a_{14} x y+a_{15} x z+a_{16} y z+a_{17} x^{2}+a_{18} y^{2}+a_{19} z^{2} \\
a_{20}+a_{21} x+a_{22} y+a_{23} z+a_{24} x y+a_{25} x z+a_{26} y z+a_{27} x^{2}+a_{28} y^{2}+a_{29} z^{2}
\end{array}\right) .
$$

The deformation parameters $a_{l k}$ pertaining to the quadratic polynomial $F$ are estimated using the following definition of pairwise discrepancy. For each 


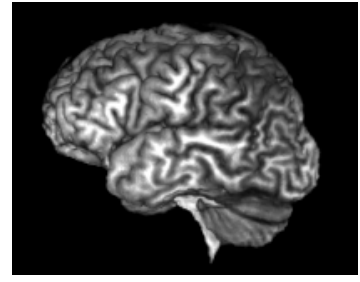

a) MR image

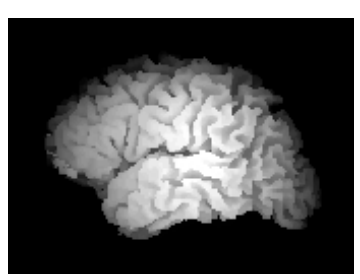

b) white matter

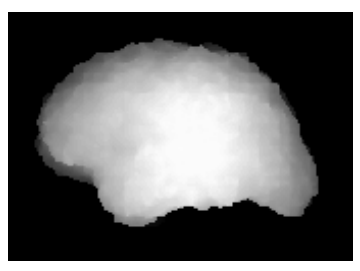

c) morphological closure

Fig. 2. Extracting a surface for subsequent warping

node $p_{i} \in \mathbb{R}^{3}, i=1, \ldots, n$ in one data set, we select the corresponding node $q_{i}$ in the model data set. The corresponding node must have the same basin label and it must be the node whose Euclidean distance $\left\|q_{i}-p_{i}\right\|$ from $p_{i}$ is minimal. The deformation parameters $a_{l k}$ are then chosen such that the following term is minimized:

$$
\sum_{i=1}^{n}\left(q_{i}-F\left(p_{i} ; a_{l k}\right)\right)^{2} .
$$

The minimization was performed using Powell's optimization method ([15]). We selected the model brain by computing pairwise discrepancies between all data sets, and choosing the one whose average discrepancy to all other brains was the least in this group.

\section{Experiments}

\subsection{Results of Spatial Normalization and Averaging}

We applied the spatial normalization to the co-registered zmaps of our 10 test subjects. Comparing the effects of the sulcal basin normalization with the simple linear normalization, we found that the sulcal basin normalization does indeed decrease the inter-subject variance of the zmaps.

Let $z_{j}^{i}$ denote the $j$ th voxel in the zmap of the $i$ th subject, and let

$$
\mu_{j}=\frac{1}{10} \sum_{i=1}^{10} z_{j}^{i}, \quad \sigma_{j}^{2}=\frac{1}{10-1} \sum_{i=1}^{10}\left(\mu_{j}-z_{j}^{i}\right)^{2}
$$

denote the inter-subject average and standard deviation at each voxel. When using a simple linear normalization, we found that the variance $\sigma_{j}$ across subjects at each voxel is larger on average than when using the sulcal basin normalization. The mean standard deviation $\sum_{j=0}^{n} \sigma_{j}$ using linear normalization was 1.233 $(\sigma=0.776)$ compared to $1.187(\sigma=0.776)(p<0.0001, t=51.28)$.

When performing a voxel-by-voxel comparison of inter-subject variance we found that 54.8 percent of the voxels had a larger inter-subject variance when 


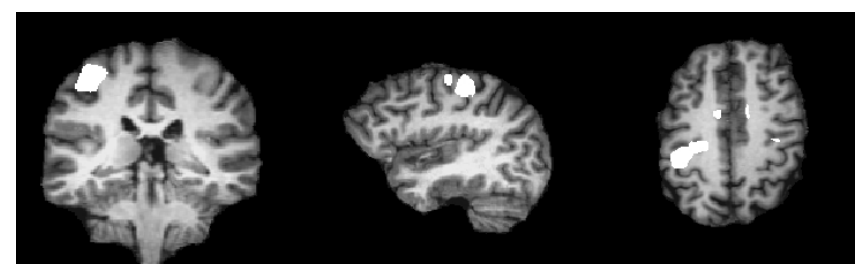

Fig. 3. Activation areas thresholded at $\mathrm{z}=2.25$ and superimposed on an anatomical MR data set.

using linear instead of sulcal basin normalization. Figure 3 shows the zmap averaged across subjects after non-linear normalization and superimposed onto one anatomical data set. It was thresholded at $z=2.25$.

Since the inter-subject variance is decreased by spatial normalization, the functional activation areas become more pronounced and more easily identifiable. In our context, activation areas are defined as connected components of the thresholded zmap. Table 1 shows two listings of activation areas obtained after linear and non-linear normalization.

\begin{tabular}{|l|rrc|}
\hline & size & sum & location \\
\hline $\mathrm{a}$ & 942 & 3102.40 & $(-9,-12,53)$ \\
$\mathrm{b}$ & 857 & 2559.49 & $(-39,-34,49)$ \\
& 2 & 5.51 & $(-45,-37,44)$ \\
$\mathrm{d}$ & 23 & 65.02 & $(-21,-24,42)$ \\
$\mathrm{c}$ & 877 & 2670.00 & $(0,-78,-15)$ \\
& 40 & 112.83 & $(-12,-85,-17)$ \\
& 3 & 8.29 & $(-14,-90,-15)$ \\
\hline & 2744 & 8523.54 & \\
\hline
\end{tabular}

a) linear normalization

\begin{tabular}{|l|rrc|}
\hline & size & sum & location \\
\hline $\mathrm{a}$ & 874 & 2877.10 & $(-10,-10,53)$ \\
$\mathrm{b}$ & 1221 & 3772.57 & $(-41,-33,49)$ \\
& 12 & 33.58 & $(-42,-22,52)$ \\
$\mathrm{a}$ & 23 & 64.74 & $(-11,0,46)$ \\
$\mathrm{d}$ & 32 & 90.38 & $(-22,-24,42)$ \\
& 3 & 8.32 & $(-28,-26,42)$ \\
$\mathrm{c}$ & 767 & 2389.69 & $(0,-78,-14)$ \\
& 1 & 2.75 & $(7,-89,-14)$ \\
\hline & 2933 & 9239.14 & \\
\hline
\end{tabular}

b) sulcal basin normalization

Table 1. Listing of activation areas with $\mathrm{z}$-values thresholded at $\mathrm{z}=2.75$. Activation sizes are given in $\mathrm{mm}^{3}$. The location is given as Talairach-Fox coordinates of the voxel with the highest $\mathrm{z}$ value. The sum represents the sum over all z-values within the area. Note that by using non-linear normalization the total sum increases by about 9 percent (from 8523.54 to 9239.14). Corresponding areas are labelled by a letters a,b,c,d in both tables. For some smaller areas correspondences could not be identified.

\subsection{Results of Group Averages Not Using Stereotactic Coordinates}

In the previous section, we have described a method of obtaining group averages using spatial normalization. However, the main advantage of the sulcal basin model is that is allows to perform group averages using anatomical locations rather than stereotactic coordinates. 
Table 2 lists the 38 basin locations and the average functional activations found in each basin. This time however, the average is obtained by summing across corresponding basins of all subjects, not across corresponding locations in the stereotactic coordinate space. The activation level of a basin (within one subject) is defined as the mean $z$-score within this basin. To determine whether a significant level of activation is present in a basin, we average activation levels across subjects and perform a $t$-test against the null hypothesis that no activation is present in the basin. In this experiment, we found a significant level of activation $(p<0.05)$ in 10 basins.

The important point here is that activations are now averaged in an anatomically meaningful manner, and the result is listed in a way that is immediately interpretable by a specialist. The results listed in table 1 correspond to the results listed in the table 2 (with the exception of right hemispheric and median activations). For instance, area "a" corresponds to basins 4,14. Note that due to the thresholding of $z$-values inherent in table 1 , one major activation area (basin 24) is missing altogether in table 1 as the $z$-scores were just below the threshold.

\begin{tabular}{|r|cl|}
\hline basin & activation level & \multicolumn{1}{c|}{$p$} \\
\hline 2 & 0.732 & 0.064 \\
3 & 1.096 & $0.002 *$ \\
4 & 1.103 & $0.005 *$ \\
5 & 1.291 & $0.027 *$ \\
6 & 0.495 & $0.018^{*}$ \\
7 & 0.193 & 0.160 \\
8 & 0.253 & 0.233 \\
9 & -0.410 & $0.023 *$ \\
10 & -0.065 & 0.416 \\
11 & 0.065 & 0.450 \\
12 & 0.098 & 0.451 \\
13 & -0.857 & 0.117 \\
14 & 0.533 & $0.042 *$ \\
15 & -0.097 & 0.390 \\
16 & 0.058 & 0.396 \\
17 & -0.125 & 0.380 \\
18 & 0.855 & $0.016 *$ \\
19 & 0.586 & 0.078 \\
20 & 0.213 & 0.108 \\
\hline
\end{tabular}

\begin{tabular}{|r|cl|}
\hline basin & activation level & \multicolumn{1}{c|}{$p$} \\
\hline 21 & 0.154 & 0.288 \\
22 & 0.507 & 0.062 \\
23 & 0.472 & 0.046 \\
24 & 0.332 & 0.103 \\
25 & 0.123 & 0.295 \\
26 & 0.489 & 0.008 \\
27 & -0.111 & 0.353 \\
28 & -0.161 & 0.191 \\
29 & 0.143 & 0.107 \\
30 & 0.027 & 0.402 \\
31 & 0.605 & 0.045 \\
32 & 0.380 & 0.160 \\
33 & 0.085 & 0.362 \\
34 & 0.089 & 0.387 \\
35 & -0.049 & 0.429 \\
36 & -0.131 & 0.227 \\
37 & 0.421 & 0.082 \\
38 & 0.044 & 0.460 \\
39 & -0.348 & 0.237 \\
\hline
\end{tabular}

Table 2. Listing of average functional activations levels across 10 subjects. Basins with significant levels of activations $(p<0.05)$ are marked by a star. The $p$-values resulted from a $t$-test against the null hypothesis that the activation level is zero. Only basins of the left lateral hemisphere are listed. The basin labels correspond to the labels displayed in figure 1. 


\section{Discussion}

We have introduced a new approach to spatial normalization and inter-subject averaging using the sulcal basin model. The prime advantage is that this model allows inter-subject comparisons on the basis of the individual anatomy rather than on a somewhat arbitrary stereotactic coordinate space. Thus, image warping might not even be necessary for inter-subject averaging as averaging can be performed using the sulcal basins instead of voxels in some coordinate space.

This new approach offers three advantages over the standard spatial normalization and pixel-wise average approach. Firstly, in averaging across sulcal basin locations instead of voxels in stereotactic coordinate space, it is guaranteed that truly corresponding entities are matched and averaged. Secondly, the results are displayed in a way that is much more useful to the specialist as it is based on an established neuro-anatomical vocabulary. A third advantage is that this method does not depend on any form of thresholding of $z$-values as is required by standard averaging methods. Thresholding of significance values is often quite arbitrary and might lead to misinterpretations. Using sulcal basins, we can directly report the presence or absence of an activation in a basin together with a significance level.

The fact that a spatial normalization based upon sulcal basins reduces the variance in the zmap-average is evidence that this model is indeed anatomically and functionally valid although further proof is warranted. The fact that the improvement in zmap-variance is only moderate is not surprising. Had we obtained drastically different results, then we would have to disbelieve either our previous results obtained by the standard linear normalization method, or be would have to doubt our new results.

The spatial normalization using second-order polynomials is a somewhat crude approach and was introduced primarily for the purpose of demonstrating the validity of the sulcal basin concept. Other methods of using sulcal basins as landmarks for warping are conceivable and will be tested in the future.

\section{Acknowledgments}

We would like to thank Dr. Volker Bosch for valuable discussions about the statistical aspects of this paper, and Dr. Stefan Pollman for providing the fMRI data.

\section{References}

1. J.W. Belliveau, D.N. Kennedy, R.C. McKinstry, B.R. Buchbinder, R.M. Weisskopf, M.S. Cohen, J.M. Vevea, T.J. Brady, B.R. Rosen. Functional mapping of the human visual cortex by magnetic resonance imaging. Science, 254:716-719, 1991. 489

2. G.E. Christensen, R.D. Rabbitt, M.L. Miller. Deformable templates using large deformation kinematics. IEEE Transactions on Image Processing, 5(10):1435-1447, 1996. 491 
3. P.T. Fox. Spatial normalization origins: objectives, applications, and alternatives. Human Brain Mapping, 3:161-164, 1995. 491

4. K.J. Friston, J. Ashburner, C.D. Frith, J.B. Poline, J.D. Heather, and R.S.J. Frackowiak. Spatial registration and normalization of images. Human Brain Mapping, 2:165-189, 1995. 491

5. G.Lohmann, D.Y.v.Cramon. Automatic detection and labelling of the human cortical folds in magnetic resonance data sets. In ECCV (European Conference on Computer Vision), Freiburg,Germany, June 2-6 1998. 489

6. G.Lohmann, D.Y.v.Cramon. Automatic labelling of the human cortical surface using sulcal basins. Medical Image Analysis, (submitted), 1998. 489

7. G.Lohmann, D.Y.v.Cramon. Sulcal basins and sulcal strings as new concepts for describing the human cortical topography. In IEEE workshop on biomedical image analysis, Santa Barbara, CA, June 1998. 489, 492

8. F. Kruggel, G.Lohmann. BRIAN (Brain Image Analysis) - a toolkit. In CAR 96, Paris, June 1996. 490

9. F. Kruggel, G.Lohmann. Automatical adaptation of the stereotactical coordinate system in brain MRI data sets. In J. Duncan, editor, Int. Conf. on Information Processing in Medical Imaging (IPMI 97), Poultney, Vermont, USA, June 9-13 1997. 490

10. J.L. Lancaster, T.G. Glass, B.R. Lankipalli, H. Downs, H. Mayberg, P.T. Fox. A modality-independent approach to spatial normalization of tomographic images of the human brain. Human Brain Mapping, 3(3):209-223, 1995. 491

11. G. Lohmann. Registration of functional mri data to $3 \mathrm{~d}$ structural $\mathrm{mr}$ reference images. Technical report, Max-Planck-Institute of Cognitive Neuroscience, Leipzig, Germany, 1998. 491

12. J.B.A. Maintz, M.A. Viergever. A survey of medical image registration. Medical Image Analysis, 2(1), March 1998. 491

13. J.C. Maziotta, A.W. Toga, A. Evans, P. Fox, J. Lancaster. A probabilistic atlas of the human brain: theory and rationale for its development. Neuroimage, 2:89-101, 1995. 490, 491

14. S. Pollmann R. Weidner, H.J. Müller, D.Y. von Cramon. Brain areas involved in visual dimension weighting. In 5th International Conference on Functional Mapping of the Human Brain (submitted), 1999. 490

15. W.H. Press, S.A. Teukolsky, W.T. Vetterling, B.P. Flannery. Numerical Recipies in C. Cambridge University Press, 2nd Edition, 1992. 493

16. S. Sandor, R. Leahy. Surface-based labeling of cortical anatomy using a deformable atlas. IEEE Transactions on Medical Imaging, 16(1):41-54, Feb. 1997. 491

17. G. Subsol, J-P Thirion, and N. Ayache. A General Scheme for Automatically Building 3D Morphometric Anatomical Atlases: application to a Skull Atlas. Medical Image Analysis, 2(1), March 1998. 491

18. J.-P. Thirion. Image matching as a diffusion process: an analogy with Maxwell's demons. Medical Image Analysis, 2(3), 1998. 491

19. P. Thompson, A.W. Toga. A surface-based technique for warping three-dimensional images of the brain. IEEE Transactions on Medical Imaging, 15(4):402-417, 1996. 491

20. A.W. Toga. Brain Warping. Academic Press, San Diego, CA, 1999. 491 